\title{
Prosthetic Application Using Tongue and Robotic Arm
}

\author{
R.V.Shinde ${ }^{1}$, S.S.Patil ${ }^{2}$ \\ Research Scholar, Rajarambapu Institute of Technology, Sakharale, Islampur, Sangli (M. S.), India ${ }^{1}$ \\ Assistant Professor, Rajarambapu Institute of Technology, Sakharale, Islampur, Sangli (M. S.), India ${ }^{2}$
}

\begin{abstract}
In recent days of science and technology, the technology is lagging back in the event of prosthetic appliances. It is necessary to boost the appliances for physically challenged persons. The techniques such as for instance ITCS (Inductive Tongue Control System) and EMG (Electromyogram) are utilized to design the system and provide financial support to physically challenged persons. Using ITCS and EMG a grasp or keyboard is operated by physically challenged persons. So that they can work with easy operations in day to day life and they can be supported financially. In this paper we review the current research in prosthetic devices for physically challenged people, focused on system to carry out daily easy operations using prosthetic arm for different types of grasps activated by sensors placed in mouth of physically challenged persons.
\end{abstract}

Keywords: ITCS (Inductive Tongue Control System), EMG (Electromyogram), mouthpiece unit, Activation unit.

\section{INTRODUCTION}

Today's researchers are greatly working in field of prosthesis for development of physically challenged people. Lots of physically challenged persons can't perform day-to-day easy operations. To overcome this they can be provided with such systems. This defines prosthetic application from the title. The prosthetic application can be thus defined as "a process of replacing physically challenged body part with artificial device for daily easy operations." This system will allow people to utilize keyboard for personal computers or laptops. This can also offer some financial support to such challenged people in small or large scale industries by performing some work. To execute these operations in this technique a artificial robotic hand should be positioned on physically challenged person hand. This robotic arm or robotic hand is controlled by tongue and electrodes on hand.

To overcome above explained problem for physically challenged persons and to construct these overall system two techniques can be used. Following are the names of two techniques:

1. ITCS (Inductive Tongue Control System)

2. EMG(Electromyogram)

Previously researcher's done the comparison of both of these approaches for same application. They figured ITCS is really a bit faster than EMG. However for this system both techniques are used. This system can also be built using single Electromyogram technique. But it has many disadvantages hence to overcome this all Electromyogram can be used in combination with ITCS technique. This system is dual modal control scheme in which the robotic arm is controlled using ITCS and EMG both techniques. 
anatomy part is physically challenged or inoperative, it is replaced. Rather than replacing it, it can use prosthetic system to create that specific body part operative.

\section{III.ITCS (INDUCTIVE TONGUE CONTROL SYSTEM)}

This technique used for this system is used to choose a specific kind of grasp. To select grasp various kinds of sensors are used. This technique includes two units as follows:

\section{Mouthpiece unit. \\ 2. Central unit}

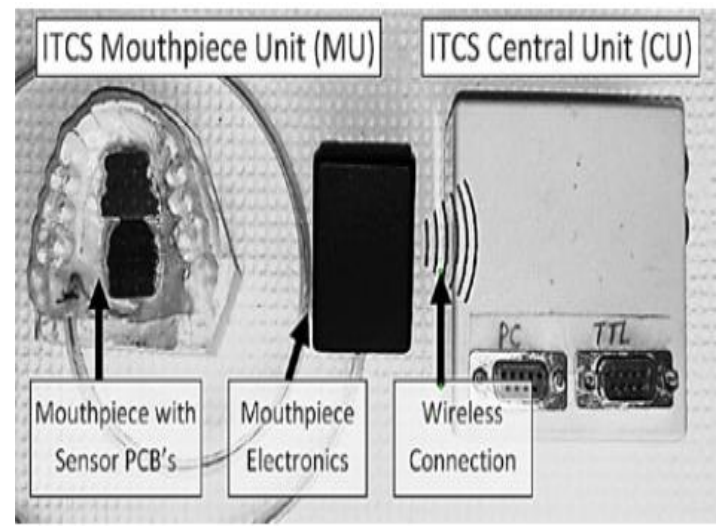

Fig.1 Inductive tongue control system unit

\section{III (1) MOUTHPIECE UNIT:}
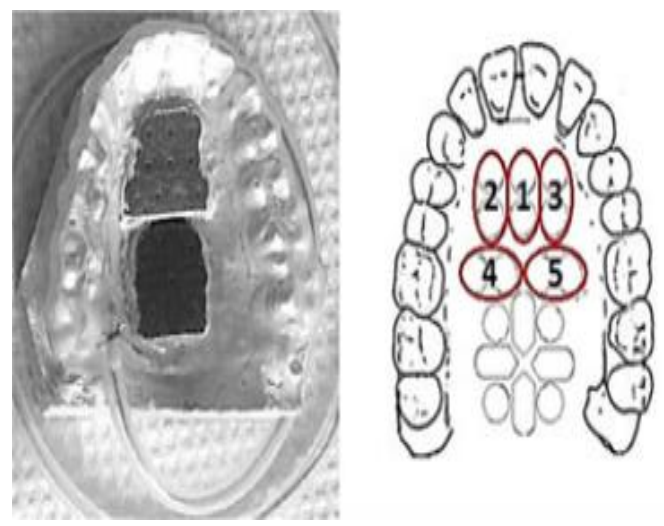

Fig.2 .Mouthpiece Unit

In this mouthpiece unit it includes two circuit boards. Both of these boards are positioned in mouth. To position the amount of sensors in mouth the measurement of upper palatal is taken. This measurement is performed by process accompanied by dentist during root canal. Previously this technique was used for wireless keyboard system. Within In this system number of sensors providing different grasps are positioned in upper palatal of mouth. Out of two printed circuit boards one includes wireless charging circuitry and other consists of sensors useful for grasps. This sensors are in form of coils. These sensors operate on the principle of Faraday's law.
As these sensors uses coil they are called as inductive sensors. These sensors placed in mouth upper palatal are activated using activation unit shown below.

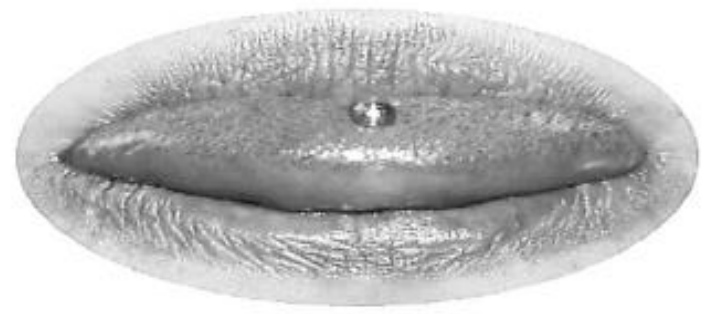

Fig. 3. Activation Unit

The above mentioned figure shows the activation unit used to activate the inductive sensors. This unit has diameter of $4 \mathrm{~mm}$ and height of $2 \mathrm{~mm}$. This activation is composed of dental alloy. This unit is placed on tongue using tissue glue which does not cause any harm to tongue skin.

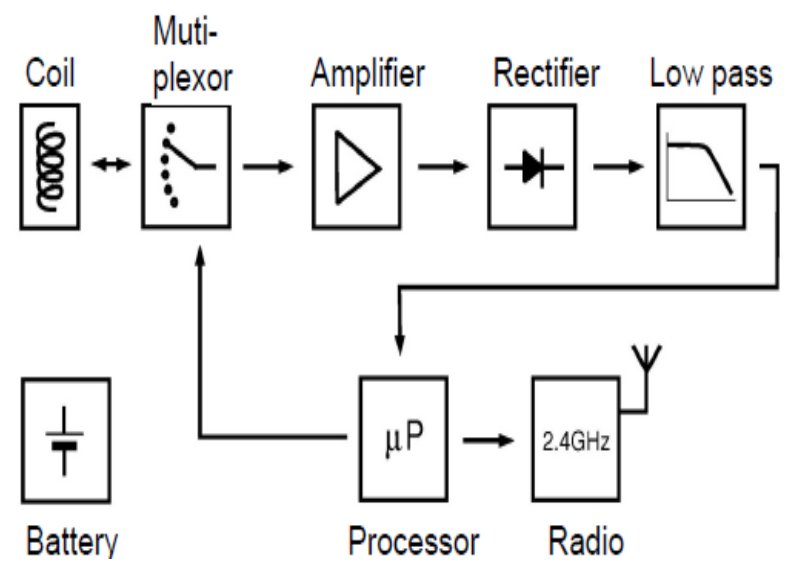

Fig.4. System from mouth

- The sensors from mouth have fixed output voltage range.

- These signals according to activation are multiplexed.

- $\quad$ These amplified signals are rectified and passed through low pass filter.

- $\quad$ The rectified signal is provided to microprocessor such that it provides sampled version of signal.

- The sampled form of signal is transmitted wirelessly to central unit for further processing.

\section{III (2) CENTRAL UNIT:}

The central unit comprises of microprocessor and receiver. It receives the signals originating from transmitter placed in mouth. It guesses the activated sensor by comparing the amplitude levels of sensors. This unit is positioned on the shoulder of user since it becomes comfortable to an individual to operate the system. 


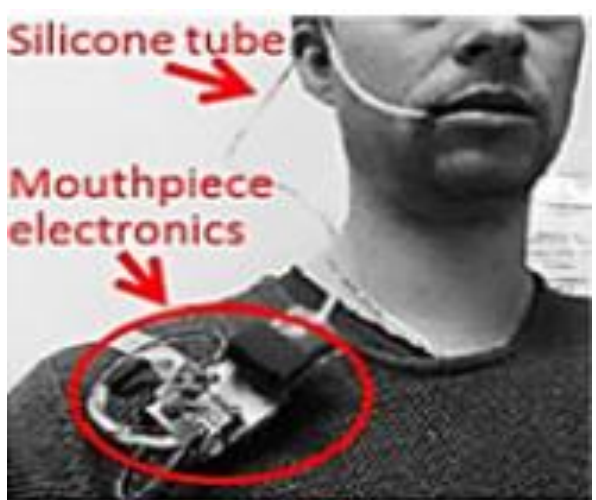

Fig .5 .Central Unit

The connection of mouthpiece electronics with sensors is performed with silicon tube shown above. Actually 18 sensors can be placed in human mouth. However in this systems 10 sensors are used. The mapping of sensors is performed in accordance with grasps required within our day to day operations. Accordingly once the activation unit touches the specific sensors the robotic arm is activated and the arm gets shaped into particular selected grasp.

\section{EMG (ELECTROMYOGRAM)}

Basically Electromyogram is technique used to test the muscle activity. This checking of muscle activity is performed using device called electromyography. In case a person has a suffering in his/her muscles then the defective muscle can be easily detected applying this technique .Accordingly where the individual have pain that body part is cleaned first. Then the needle electrode is placed in that area. There is continuous swapping of two kinds of tissues in human muscles. EMG signal is recorded means this swapping is checked. The recorded signals are accustomed to control prosthetic devices.

As explained above there is only the activation of grasp. Nevertheless the opening or closing of the prosthetic arm is completed using EMG technique.

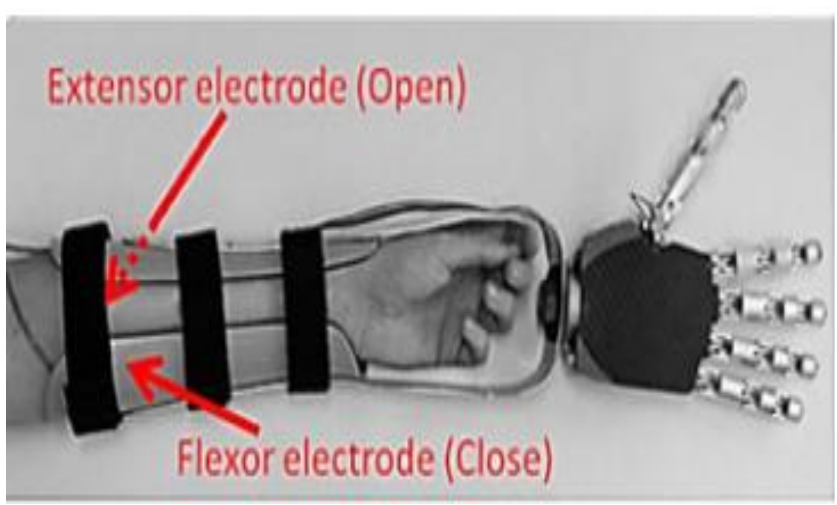

Fig.6. Electromyogram System
In this system the opening and closing of particular grasp is performed in accordance with electrodes. The outer lining electrodes are triggered and the arm operates. The triggering of surface electrodes is dependent upon contraction of muscle. The contraction of muscle is determined by length and tension over muscles. In this contraction technique both proteins actin and myosin swaps and the recorded signal is the swapping of this to proteins.

The central unit of ITCS receives output of both EMG and ITCS input signals. This in turn controls the opening and closing of prosthetic hand. As the key surface electrode of EMG was triggered, the output signal was amplified, filtered and rectified and then employed for comparison. This analog signal is then transformed into digital and then provided to central unit of ITCS system. To offer this digital signal to central unit, the central unit is provided with two input/output channels.

Out of two electrodes which are flexor and extensor, the electrode which includes it's voltage significantly more than or corresponding to active threshold point, that electrode is prioritized and accordingly opening or closing action takes place. If the voltage across flexor is significantly more than or equal to 'active threshold' point and the voltage across extensor electrode is close to 'inactive threshold' point, this causes the closing action of grasp of prosthetic arm and vice versa.

\section{WORKING OF OVERALL SYSTEM}

The various sensors placed in mouth are activated regarding required grasp.

- $\quad$ These sensors are activated using activation unit positioned on tongue.

- This activation is provided to central unit of ITCS technique placed on shoulder through wireless transmitter placed in mouth.

- The receiver on shoulder receives this signal and the robotic arm is pre shaped into activated grasp.

- $\quad$ According to activation of grasp the extensor muscle or wrist flexor contraction takes place. This is completed under EMG technique.

- The process of contraction activates extensor or flexor electrode.

- $\quad$ Active threshold voltage and non active threshold voltage are set at fixed levels.

If the flexor voltage greater than or equal to active threshold voltage then closing of robotic hand of particular grasp and required fingers is carried out.

- If the extensor voltage is greater than or equal to non active threshold voltage then opening of robotic hand of particular grasp and required fingers is carried out.

The five grasp used in this system are: 
[1] Precision pinch

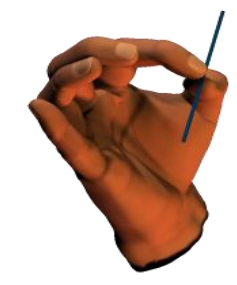

[2] Lateral pinch

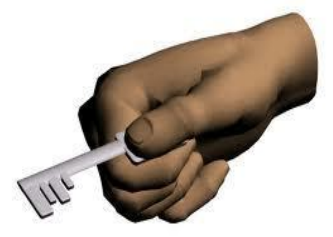

[3] Diagonal volar grasp

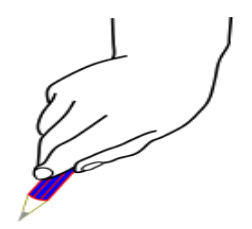

[4] Transversal volar grasp

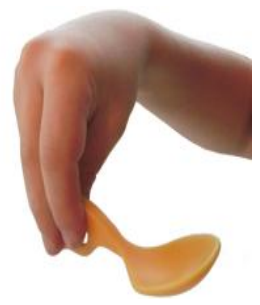

[5] Tripod pinch

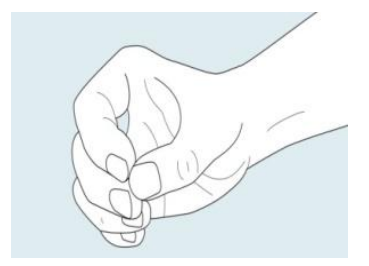

The above figures shows the grasps for this system. Other types of grasps can also be used by adding some more sensors to system.

\section{VI.CONCLUSION}

A system with dual techniques ITCS and EMG has been built for control of robotic arm. The system can be built utilizing the two techniques alone. Through experimentation EMG technique is straight forward than ITCS. As ITCS technique consist its system part in mouth, this causes problem during saliva of person. To test the 